\title{
Is There a "Hidden" Effect of Treadmill Walking on Step Characteristics, in Children With Cerebral Palsy and Typically Developed Controls?
}

\author{
Michal Katz-Leurer $\stackrel{\text { a, }}{\text {, }}$ Hemda Rotem ${ }^{\mathrm{b}}$, Shirley Meyer
}

\begin{abstract}
Background: To examine the immediate effect of treadmill walking, on gait variability among children with cerebral palsy and $\mathrm{TD}$ age matched controls.

Methods: Eleven children with eerebral palsy and sixteen TD controls participated in this study. Ages were $7-13$ years. Step time and length variability were assessed by an electronic walkway prior to and after treadmill walking for six minutes.
\end{abstract}

Results: When walking on level ground prior to the treadmill session children with CP walked with significantly increased step time and step time and length variability and significant reduced step length compared to the TD subjects. Treadmill walking reduced walking velocity, increased step time and decreased step length variability significantly among children with $\mathrm{CP}$.

Conclusions: Walking on a treadmill at a fixed gait velocity impact gait characteristics among children with $\mathrm{CP}$, with possible advantageous effect on step length variability.

Keywords: Gait; Cerebral palsy; Step variability; Treadmill

\section{Introduction}

Walking disturbances is one of the main functional disabili-

Manuscript accepted for publication August 17, 2012

${ }^{a}$ Tel Aviv University, physiotherapy department, Israel

bAlyn Children's Orthopedic Hospital and Rehabilitation Center, Jerusalem, Israel

cAlyn Children's Orthopedic Hospital and Rehabilitation Center, Jerusalem, Israel

${ }^{\mathrm{d}}$ Corresponding author:Michal Katz-Leurer, Tel Aviv University, physiotherapy department, Israel.Email: michalkz@post.tau.ac.il

doi: http://dx.doi.org/10.4021/jnr132w ties of children with Cerebral Palsy (CP). Treadmill training is a popular walking training method, based on current theories of motor learning [1]. Physical effects of therapy and task specific practice, as well as neuroplasticity post brain damage, are believed to play a role in the mechanics of treadmill training in patients with brain damage [2].

Studies have evaluated the effect of treadmill walking on children with $\mathrm{CP}$ and found an improvement in gait speed, balance during walking and in term of aerobic improvement in mind, children with CP may improve endurance capacities due to the cardio-respiratory adaptation to treadmill walking [3].

Gait velocity on a treadmill is constant and repetitive. A repetitive sequence of gait (as on the treadmill) may activate CNS neural circuits that mediate central patterns of motor control and the resultant rhythmic motor commands and muscle activation might lead to a decrease in variability in gait (increase in "automatism") [1]. A treadmill also imposes an external pace that is mediated through proprioceptive and vestibular receptors which generate repetitive sensory input to the central nervous system [2].

Frenkel-Toledo et al reported that among healthy adults, walking on a treadmill reduces step variability [4]. Katz-Leurer et al Bescribed that among typically developed (TD) children, walking on a treadmill reduces step variability while no effect observed among children post traumatic brain injury [5]. It has been described before that as compared to typically developed controls children with CP demonstrate increased step variability parameters while walking [6]. High level of step variability, found to be associated with poor balance in neurological patients $[7,8]$.

It might be that in additional to the known benefits of treadmill training to walking speed and aerobic abilities, walking on a treadmill has a further effect for children with CP. The aim of the present study was to examine the immediate effect of treadmill walking on step variability in children with cerebral palsy and typieally developed (TDł controls. Such an effect is a kind of memory activity so, as on different modality of memory it might be productive to look on immediate effect versus median/long effect (i.e. Short-term memory versus Long-term memory). 
Table 1. Descriptive Data of Children With CP and TD Controls

\begin{tabular}{llll}
\hline & $\begin{array}{c}\text { TD } \\
(\mathbf{n}=\mathbf{1 6})\end{array}$ & $\begin{array}{c}\mathbf{C P} \\
(\mathbf{n}=\mathbf{1 1})\end{array}$ & p value \\
\hline Demographic & $9.9 \pm 1.3$ & $9.2 \pm 2.0$ & 0.93 \\
Age (yrs) & $9(56.3)$ & $6(54.5)$ & 0.10 \\
Gender (male-N(\%)) & $8.5 \pm 32.0$ & $8.5 \pm 26.3$ & 0.005 \\
Anthropometric & $10.1 \pm 142.5$ & $15.1 \pm 127.8$ & 0.34 \\
Weight (kg) & $3.4 \pm 16.3$ & $1.7 \pm 15.6$ & \\
Height (meter) & & \\
BMI (kg/meter $\left.{ }^{2}\right)$ & & & \\
\hline
\end{tabular}

Values are mean \pm standard deviation (SD)/number (percents)

Abbreviations: BMI, body mass index

\section{Methods}

\section{Subjects}

Eleven children with CP (seven were classified as hemiplegic and 4 as diplegic) who were either outpatients or former patients of the Alyn Children's Rehabilitation Hospital in Jerusalem, Israel, were selected according to the following criteria: (1) GMFCS $<3$ [9] (2) ages 7 to 13 years. Children were excluded if they (1) had used a treadmill during the 3 months prior to the study (2) received botulinum toxin for spasticity management or had undergone orthopedic surgery in the past six months (3) were unable to follow simple instructions. Sixteen TD children matched for age served as controls. Demographic, anthropometric and clinical characteristics of the children with CP and TD groups are summarized in Table 1. Informed consent was obtained from each participant and his parents before inclusion in the study. The study protocol was approved by the hospital's institutional ethical committee.

\section{Study protocol}

The study was carried out in a single session that involved a pre-treadmill walking assessment, 6-min treadmill training, and a post-treadmill walking assessment. Gait parameters were recorded on the electronic mat (the Gaitrite system) with each subject walking at a comfortable pace. Walking on the motorized treadmill was set to each subject's self-selected comfortable speed as assessed during the initial evaluation on the Gaitrite. All study subjects were asked to hold onto the handrails (sidebars) during treadmill walking. The six counted minutes on the treadmill began after 30 seconds of gradually increasing the treadmill speed to the desired speed. Assessments on the Gaitrite were made immediately before and immediately after treadmill walking.

\section{Tests and measurements}

Anthropometric measurements included body weight and height. Body mass index (BMI) was calculated from weight $\bullet$ height ${ }^{-2}\left(\mathrm{~kg}^{\prime} \mathrm{m}^{-2}\right)$.

Gait - Parameters of gait were recorded using a pressure sensitive mat, the "Gaitrite1" system [(Gaitrite ${ }^{\circledR}$, CIR Systems Inc., Clifton, NJ, USA)] which is an electronic walkway that automates the collection of spatial and temporal parameters of gait. The validity of the Gaitrite system has been supported by studies in adults [10]. Thorpe et al (2006) reeently showed that the Gaitrite ${ }^{\circledR}$ system is a reliable method of measuring spatiotemporal gait parameters in children as well [11].

The children were asked to walk along the mat at their regular speed. "Stop" signs were positioned on the floor 2 meters beyond each end of the walkway, providing visual feedback to subjects. Each cycle of walking was about 7.66 m', of which 3.66 m' were on the mat. Each subject completed three sequential cycles of walking for a total of almost 11 meters on the mat. Testing was performed with shoes and orthotics when needed. All temporal and spatial gait parameters were calculated utilizing the software of the Gaitrite system using pre-programmed definitions, calculations and data from the three cycles.

Parameters analyzed: 1) Step length was measured on the horizontal axis of the walkway from the posterior point of the current footfall to the posterior point of the previous footfall of the opposite foot. 2) Step time was selected as the temporal gait characteristic since it has been widely studied by other investigators. Step time was measured as the time 


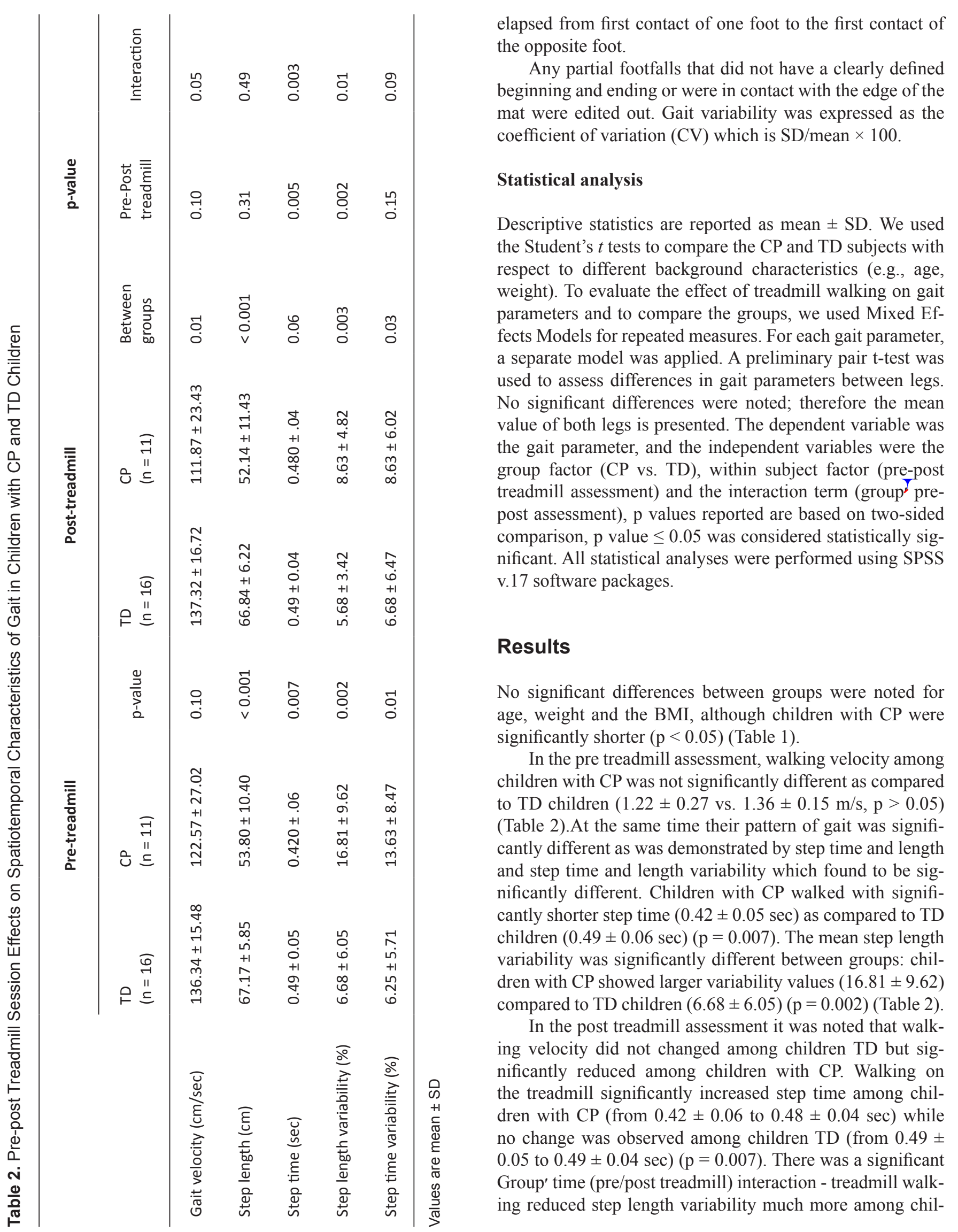


dren with $\mathrm{CP}$ (from $16.81 \pm 9.62$ to $8.63 \pm 4.82$ ) as compared to TD children (from $6.68 \pm 6.05$ to $5.68 \pm 3.42)(\mathrm{p}=0.01)$.

\section{Discussion}

The main finding of this study was that the treadmill walking significantly reduces step variability among children with CP. This finding can point that such activity does have physiological effects, as well as it might be useful to use this training for clinical practical in the goal to reduce the variability during walking in children with CP. It support the assumption that walking on a treadmill (i.e. "force" a constant speed gait) even for several minutes can induce changes in CNS activity at least for short period (as is demonstrated by it's effect on step variability reduction). It might be possible to induce from these findings that a constant pace walking (a sequence of repetitively motor function) have an impaction on changing the "automatic control" which regulate gait. If this estimation is true it might imply that walking on the treadmill activates neural circuits that have impact on "Automatism" i.e. influence changes of motor sequence activity. It has been suggested before that the basic rhythm of human locomotion is generated by the central pattern generators which work together with sensory mechanisms. The immature locomotion pattern of infants mostly reflects these spinal mechanisms [12]. Supra-spinal control above these spinal mechanisms increases with age in TD children while children with CP maintain with this immature pattern, somewhat due to injury of supraspinal motor systems. If this is true it might be speculated that in children with CP the damage CNS did not impaired the ability to trigger better automatism activity. Or possibly, walking on the treadmill might reduce the degrees of freedom and as a result helps to reduce variability. In other words, the effect of walking on a treadmill might stimulate and facilitate "inner rhythm" mechanisms which control the pattern of normal walking. A possible explanation that such effect was not seen among TD children is that their automatic control was activated near "the ceiling potential" - i.e. their initial step variability was small. In healthy adults, these stride-to-stride fluctuations are relatively small and the coefficient of variation of many gait parameters (e.g., gait speed, stride time) is on the order of just a few percent [13].

Previous studies among children with CP describe the potential long term beneficial effect of treadmill walking on gait speed and endurance. This study presents the immediate effect of treadmill walking on step variability and as such presents only the "after effect". In other neurological damage as traumatic brain injury, increased step variability associate with poor balance abilities. Possibly a longer treadmill session or frequent treadmill sessions will improve strength and endurance and then one might reassess the effect of treadmill walking on step variability.
These findings are even more powerful based on Yamasaki et al [14] and others $[15,16]$ which described a Ushaped relationship between step length variability and gait speed when healthy subjects walked on a treadmill [14]. Minimum values of variability were obtained at the comfortable walking speed (CWS) and increased when subjects walked slower or faster than the CWS. It has been suggested that minimal variability of step length occurs at the CWS because, mechanically, the most efficient gait occurs at this speed and metabolic energy expenditures are at a minimum. In our study, children were instructed to walk at the preferred, most comfortable walking speed during the session; after the treadmill session they walked more slowly, one might assume that they should presents higher variability parameters while the opposite has happened.

Other external sensory cueing may help to augment the deficient internal cueing in these children and improve gait. External cueing using rhythmic auditory stimulation or visual cueing are other possible alternatives for imposing an external pace thereby allowing children with $\mathrm{CP}$ to focus more attention on the gait.

\section{References}

1. Van de Crommert HW, Mulder T, Duysens J. Neural control of locomotion: sensory control of the central pattern generator and its relation to treadmill training. Gait Posture. 1998;7(3):251-263.

2. Mathiowetz V, Haugen JB. Motor behavior research: implications for therapeutic approaches to central nervous system dysfunction. Am J Occup Ther. 1994;48(8):733745.

3. Willoughby KL, Dodd KJ, Shields N. A systematic review of the effectiveness of treadmill training for children with cerebral palsy. Disabil Rehabil. 2009;31(24):19711979.

4. Frenkel-Toledo S, Giladi N, Peretz C, Herman T, Gruendlinger L, Hausdorff JM. Treadmill walking as an external pacemaker to improve gait rhythm and stability in Parkinson's disease. Mov Disord. 2005;20(9):11091114.

5. Katz-Leurer M, Rotem H, Keren O, Meyer S. The effect of variable gait modes on walking parameters among children post severe traumatic brain injury and typically developed controls. NeuroRehabilitation. 2011;29(1):45-51.

6. Descatoire A, Femery V, Potdevin F, Moretto P. Step-tostep reproducibility and asymmetry to study gait autooptimization in healthy and cerebral palsied subjects. Ann Phys Rehabil Med. 2009;52(4):319-329.

7. Balasubramanian CK, Neptune RR, Kautz SA. Variability in spatiotemporal step characteristics and its relationship to walking performance post-stroke. Gait Posture. 
2009;29(3):408-414.

8. Katz-Leurer M, Rotem H, Lewitus H, Keren O, Meyer S. Relationship between balance abilities and gait characteristics in children with post-traumatic brain injury. Brain Inj. 2008;22(2):153-159.

9. Palisano RJ, Cameron D, Rosenbaum PL, Walter SD, Russell D. Stability of the gross motor function classification system. Dev Med Child Neurol. 2006;48(6):424428.

10. McDonough AL, Batavia M, Chen FC, Kwon S, Ziai J. The validity and reliability of the GAITRite system's measurements: A preliminary evaluation. Arch Phys Med Rehabil. 2001;82(3):419-425.

11. Thorpe DE, Dusing SC, Moore CG. Repeatability of temporospatial gait measures in children using the GAITRite electronic walkway. Arch Phys Med Rehabil.
2005;86(12):2342-2346.

12. Forssberg H. Ontogeny of human locomotor control. I. Infant stepping, supported locomotion and transition to independent locomotion. Exp Brain Res. 1985;57(3):480-493.

13. Hausdorff JM. Gait variability: methods, modeling and meaning. J Neuroeng Rehabil. 2005;2:19.

14. Yamasaki M, Sasaki T, Torii M. Sex difference in the pattern of lower limb movement during treadmill walking. Eur J Appl Physiol Occup Physiol. 1991;62(2):99-103.

15. Danion F, Varraine E, Bonnard M, Pailhous J. Stride variability in human gait: the effect of stride frequency and stride length. Gait Posture. 2003;18(1):69-77.

16. Sekiya N, Nagasaki H, Ito H, Furuna T. Optimal walking in terms of variability in step length. J Orthop Sports Phys Ther. 1997;26(5):266-272. 